\title{
Special values of multiple gamma functions
}

\author{
par W. DUKE et Ö. IMAMOḠLU
}

RÉsumÉ. Nous donnons une formule de type Chowla-Selberg qui relie une généralisation de l'eta-fonction à $\mathrm{GL}(n)$ avec fonctions gamma multiples. Nous présentons également quelques identités de produit infinies pour certaines valeurs spéciales de la fonction gamma multiple.

ABSTRACT. We give a Chowla-Selberg type formula that connects a generalization of the eta-function to $\mathrm{GL}(n)$ with multiple gamma functions. We also present some simple infinite product identities for certain special values of the multiple gamma function.

\section{Introduction.}

The area of a quarter of the unit circle is $\int_{0}^{1} \sqrt{1-t^{2}} d t=\frac{\pi}{4}$. Less familiar are the identities

$$
\begin{aligned}
& \int_{0}^{1} \sqrt[4]{1-t^{2}} d t=\frac{\pi}{3} \prod_{m \in \mathbb{Z}^{+}} \tanh ^{2}\left(\frac{\pi m}{2}\right) \text { and } \\
& \int_{0}^{1} \sqrt[8]{1-t^{2}} d t=\frac{\alpha \pi}{5} \prod_{m \in \mathbb{Z}^{+}} \tanh ^{2}\left(\frac{\pi m}{\sqrt{2}}\right)
\end{aligned}
$$

where $\alpha=(1+\sqrt{2})^{1 / 2}$. In terms of the gamma function $\Gamma(x)$ we have, for any $x>0$, that

$$
\int_{0}^{1}\left(1-t^{2}\right)^{x-1} d t=2^{2 x-2} \frac{\Gamma^{2}(x)}{\Gamma(2 x)}
$$

and so (1) and (2) are equivalent to special infinite products for the values $\Gamma\left(\frac{1}{4}\right)$ and $\Gamma\left(\frac{1}{8}\right)$. For instance, (1) is equivalent to

$$
\Gamma\left(\frac{1}{4}\right)=(2 \pi)^{3 / 4} \prod_{m \in \mathbb{Z}^{+}} \tanh \left(\frac{\pi m}{2}\right)
$$

after using the well known formula $\Gamma\left(\frac{1}{2}\right)=\sqrt{\pi}$.

\footnotetext{
The research of both authors were partially supported by NSF. The first author thanks ETH for providing hospitality and financial support.
} 
These facts are classical and go back, at least in principle, to Gauss and Jacobi. Simple proofs are given in $\S 4$ below using the Kronecker limit formula. This technique was introduced by Chowla and Selberg [10], who showed how to obtain more general results of this type in terms of special values of the Dedekind eta-function. This is defined for $\tau \in \mathcal{H}$, the upper half plane by

$$
\eta(\tau)=e^{\pi i \tau / 12} \prod_{m \in \mathbb{Z}^{+}}\left(1-e^{2 \pi i m \tau}\right) .
$$

Suppose that $\tau=x+i y \in \mathcal{H}$, the upper half-plane, is a root of the integral quadratic equation

$$
a z^{2}+b z+c=0
$$

where $D=b^{2}-4 a c<0$ is a fundamental discriminant. Let $\chi(\cdot)=\left(\frac{D}{\cdot}\right)$ be the Kronecker symbol, which is a Dirichlet character defined $\bmod N=$ $|D|$. Let $h$ be the class number of $\mathbb{Q}(\sqrt{D})$ and $w$ be the number of roots of unity in $\mathbb{Q}(\sqrt{D})$. The Chowla-Selberg formula [10] states that

$$
y|\eta(\tau)|^{4}=\frac{\lambda}{\pi} \prod_{r=1}^{N} \Gamma\left(\frac{r}{N}\right)^{(w / 2 h) \chi(r)}
$$

for some explicitly computable algebraic number $\lambda$.

In this article we will give a Chowla-Selberg type formula that connects a generalization $\eta_{n}$ of the Dedekind eta-function to $\mathrm{GL}(n)$ with multiple gamma functions $\Gamma_{\ell}$ for $\ell=1, \ldots k=n / 2$. More precisely, we prove in Theorem 2 that for $Q$ a positive-definite even unimodular $n \times n$ matrix we have

$$
y\left|\eta_{n}(Q)\right|^{4}=\frac{\lambda}{\pi} \prod_{\ell=1}^{k} \Gamma_{\ell}\left(\frac{1}{2}\right)^{C_{k, \ell}},
$$

where $\lambda=2^{C_{k}-2} e^{-L^{\prime}(0, f)}$ for a certain $L$-function $L(s, f)$ and $C_{k}, C_{k, \ell}$ are explicit rational numbers. Along the way we will present some simple infinite product identities like (4) for certain special values of the multiple gamma function.

\section{The multiple gamma function}

The multiple gamma functions we consider were first studied by Barnes [2]. Set $\Gamma_{0}(x)=x^{-1}$ and fix $n \in \mathbb{Z}^{+}$. As a real function, the multiple gamma function $\Gamma_{n}(x)$ can be defined as the unique positive real valued $n$-times differentiable function on $\mathbb{R}^{+}$with the property that

$$
(-1)^{n+1} \frac{d^{n}}{d x^{n}} \log \Gamma_{n}(x)
$$


is increasing and that satisfies

$$
\Gamma_{n}(x+1)=\frac{\Gamma_{n}(x)}{\Gamma_{n-1}(x)} \quad \text { and } \quad \Gamma_{n}(1)=1 .
$$

The existence and uniqueness of $\Gamma_{n}(x)$ follows from [8], which actually produces a Weierstrass product expansion for $\Gamma_{n}(x+1)^{-1}$ from these conditions that shows it to be an entire function of order $n$ with zeros at the negative integers:

(8) $\frac{1}{\Gamma_{n}(x+1)}=e^{P_{n}(x)} \prod_{k \geq 1}\left(\left(1+\frac{x}{k}\right) \exp \left(-\frac{x}{k}+\frac{x^{2}}{2 k^{2}}-\cdots+\frac{(-x)^{n}}{n k^{n}}\right)\right)^{\left(\begin{array}{c}k+n-2 \\ n-1\end{array}\right)}$,

for a certain polynomial $P_{n}(x)$ of degree $n$ (see [18, p.241] for more details). In particular,

$$
\begin{aligned}
& P_{1}(x)=\gamma x \\
& P_{2}(x)=\left(\frac{\log 2 \pi}{2}-\frac{1}{2}\right) x-\left(\frac{\gamma}{2}+\frac{1}{2}\right) x^{2} \\
& P_{3}(x)=\left(\frac{\zeta^{\prime}(-1)}{1}-\frac{\log 2 \pi}{4}+\frac{7}{24}\right) x+\left(\frac{\gamma+\log 2 \pi}{4}+\frac{1}{8}\right) x^{2}-\left(\frac{\gamma}{6}+\frac{\pi^{2}}{36}+\frac{1}{4}\right) x^{3},
\end{aligned}
$$

where $\gamma$ is Euler's constant. Clearly $\Gamma_{1}(x)=\Gamma(x)$, the usual gamma function.

It is obvious from (7) that $\Gamma_{n}(x)^{(-1)^{n-1}} \in \mathbb{Z}^{+}$for $x \in \mathbb{Z}^{+}$; for instance $\Gamma_{1}(k+1)=k$ ! and $\Gamma_{2}(k+2)^{-1}=k ! !=\prod_{\ell=1}^{k} \ell$ !. In this paper we are interested in the value $\Gamma_{n}(x)$ for $x$ half an odd integer. Our first goal is to show that the values $\Gamma_{2}\left(\frac{1}{2}\right)=1.65770324 \ldots$ and $\Gamma_{3}\left(\frac{3}{2}\right)=.95609000 \ldots$ can be evaluated as infinite products of the type occurring in (4). The following are proven in $\S 4$.

Theorem 1. We have the identities

$$
\begin{aligned}
& \text { (i) } \quad \Gamma_{2}\left(\frac{1}{2}\right)^{4}=(\sqrt{2} \pi)^{3 / 2} \prod_{m \in \mathbb{Z} \times 2 \mathbb{Z} \times 2 \mathbb{Z}}^{+} \tanh ^{2}\left(\frac{\pi|m|}{2}\right) \text { and } \\
& \text { (ii) } \quad \Gamma_{3}\left(\frac{3}{2}\right)^{-4}=(2 \sqrt{\pi})^{1 / 2} \prod_{\substack{m \in(\sqrt{2} \mathbb{Z})^{4} \times \mathbb{Z} \\
m_{5} \text { odd }}}^{+} \tanh ^{2}\left(\frac{\pi|m|}{2}\right) \text {, }
\end{aligned}
$$

where the + superscript indicates that the product is taken over some choice of $\pm m$ and $m$ is non-zero. Here $|m|$ is the usual Euclidean norm.

It should be observed that these do not follow directly from the Weierstrass product (8).

Many of the well known identities involving the gamma function have generalizations to $\Gamma_{n}$ for $n>1$, including the multiplication formula of Gauss. However, there are no known generalizations of identities for period integrals like (3). Nevertheless, special values of multiple gamma functions like those in Theorem 1 occur in some geometric quantities, for 
instance the determinants of certain Laplacians. Recall that if $\mathcal{M}$ is a compact $n$-dimensional Riemannian manifold, the Laplacian $\Delta=\Delta_{\mathcal{M}}$ (on functions) on $\mathcal{M}$ has discrete spectrum

$$
0=\lambda_{0}<\lambda_{1} \leq \lambda_{2} \leq \cdots \leq \lambda_{n} \leq \cdots
$$

Its associated spectral zeta-function

$$
Z_{\mathcal{M}}(s)=\sum_{j \geq 1} \lambda_{j}^{-s}
$$

is absolutely convergent for $\operatorname{Re}(s)$ sufficiently large and has a meromorphic continuation in $s$ to the entire $s$-plane with no pole at $s=0$ [7]. This allows one to define ${ }^{1}$ the determinant of $\Delta_{\mathcal{M}}$ to be

$$
\operatorname{det} \Delta_{\mathcal{M}}=e^{-Z_{\mathcal{M}}^{\prime}(0)}
$$

In case $\mathcal{M}=S^{n}$ is the unit sphere with the standard metric, Vardi [16] discovered that det $\Delta_{S^{n}}$ can be expressed in the form

$$
\operatorname{det} \Delta_{S^{n}}=\lambda_{n} e^{A_{n}} \prod_{\ell=1}^{n} \Gamma_{\ell}\left(\frac{1}{2}\right)^{A_{n, \ell}}
$$

for certain rational numbers $A_{n}$ and $A_{n, \ell}$ and an algebraic number $\lambda_{n}$. This result should be compared with the Chowla-Selberg formula (6) since, as follows from (25) below,

$$
\operatorname{det} \Delta_{\mathcal{M}}=y^{2}|\eta(\tau)|^{4},
$$

where $\mathcal{M}=\mathbb{C} /(\mathbb{Z}+\tau \mathbb{Z})$, a flat torus. Explicitly, $\operatorname{det} \Delta_{S^{1}}=4 \pi^{2}$ and by [14, p.321] and (14) and (15) below:

$$
\begin{aligned}
& \operatorname{det} \Delta_{S^{2}}=2^{1 / 9} e^{1 / 2} \pi^{-2 / 3} \Gamma_{2}\left(\frac{1}{2}\right)^{8 / 3}=3.19531149 \ldots \\
& \operatorname{det} \Delta_{S^{3}}=\pi^{8 / 7} \Gamma_{3}\left(\frac{3}{2}\right)^{16 / 7}=3.33885121 \ldots
\end{aligned}
$$

Thus the identities of Theorem 1 can be put in the form

$$
\begin{aligned}
& \left(\operatorname{det} \Delta_{S^{2}}\right)^{3}=\beta \pi \prod_{m \in \mathbb{Z} \times 2 \mathbb{Z} \times 2 \mathbb{Z}}^{+} \tanh ^{4}\left(\frac{\pi|m|}{2}\right) \text { and } \\
& \left(\operatorname{det} \Delta_{S^{3}}\right)^{7}=\frac{1}{4} \pi^{7} \prod_{\substack{m \in(\sqrt{2} \mathbb{Z})^{4} \times \mathbb{Z} \\
m_{5} \text { odd }}}^{+} \operatorname{coth}^{8}\left(\frac{\pi|m|}{2}\right)
\end{aligned}
$$

where $\beta=2^{11 / 6} e^{3 / 2}$.

\footnotetext{
${ }^{1}$ We write $\operatorname{det} \Delta_{\mathcal{M}}$ in place of the more proper $\operatorname{det}^{\prime} \Delta_{\mathcal{M}}$. See e.g. [9] for an exposition of the theory of determinants of Laplacians and for further references.
} 


\section{Derivatives of Dirichlet $L$-functions at non-positive integers}

Let $L(s, \chi)$ be a Dirichlet $L$-function with a Dirichlet character $\chi$ defined $\bmod N$. In this section we will give a general formula for $L^{\prime}(1-k, \chi)$, when $k \in \mathbb{Z}^{+}$, in terms of multiple gamma functions at rational arguments. This formula largely accounts for the appearance of special values of the multiple gamma function in number theory and is needed for our applications. ${ }^{2}$

Define the multiple Hurwitz zeta-function for $x>0$ and $\operatorname{Re}(s)>n$ by:

$$
\zeta_{n}(s, x)=\sum_{m_{1} \geq 0} \cdots \sum_{m_{n} \geq 0}\left(m_{1}+\cdots+m_{n}+x\right)^{-s}=\sum_{m \geq 0}\left(\begin{array}{c}
m+n-1 \\
n-1
\end{array}\right)(m+x)^{-s} .
$$

Here $\zeta_{1}(s, x)=\zeta(s, x)$ is the usual Hurwitz zeta function. In general, $\zeta_{n}(s, x)$ is easily seen to have a meromorphic continuation in $s$ to the whole $s$-plane with poles at $s=1, \ldots, n$. Also, it visibly satisfies

$$
\zeta_{n}(s, x+1)=\zeta_{n}(s, x)-\zeta_{n-1}(s, x) .
$$

Writing $\zeta_{n}^{\prime}(s, x)=\frac{\partial}{\partial s} \zeta_{n}(s, x)$, it is clear that $F_{n}(x)=\exp \left(\zeta_{n}^{\prime}(0, x)\right)$ satisfies

$$
F_{n}(x+1)=\frac{F_{n}(x)}{F_{n-1}(x)} \quad \text { and } \quad(-1)^{n+1} \frac{d^{n+1}}{d x^{n+1}} \log F_{n}(x)=n ! \zeta_{n}(n+1, x) \geq 0 \text {. }
$$

As Vardi observed in [16], the criterion (7) implies that

$$
\log F_{n}(x)=\log \Gamma_{n}(x)+R_{n}(x)
$$

for a polynomial $R_{n}(x)$ of degree $n-1$. By writing $\zeta_{k}(s, x)$ in terms of $\zeta(s-\ell+1, x)$ for $1 \leq \ell \leq k$ and then inverting these equations, Adamchik [1] found an identity for $\zeta^{\prime}(1-k, x)-\zeta^{\prime}(1-k)$ in terms of $\log \Gamma_{\ell}(x)$ for $1 \leq \ell \leq k$. His result [1, Prop. 3] easily implies that for $k \geq 0$

$$
\begin{gathered}
\zeta^{\prime}(1-k, x)-\zeta^{\prime}(1-k)=\sum_{\ell=1}^{k} P_{k, \ell}(x) \log \Gamma_{\ell}(x) \\
\text { where } P_{k, \ell}(x)=\sum_{j=1}^{\ell}\left(\begin{array}{c}
\ell-1 \\
j-1
\end{array}\right)(-1)^{j+k}(j-x)^{k-1} .
\end{gathered}
$$

By using the relation $\zeta\left(s, \frac{1}{2}\right)=\left(2^{s}-1\right) \zeta(s)$ and the obvious formula $L(s, \chi)=$ $N^{-s} \sum_{r=1}^{N} \chi(r) \zeta\left(s, \frac{r}{N}\right)$, we derive from (10) the following.

Proposition 3.1. For $k \in \mathbb{Z}^{+}$

$$
\begin{aligned}
\left(1-2^{k}\right) \zeta^{\prime}(1-k)+ & (\log 2) \zeta(1-k) \\
& =(-1)^{k} \sum_{\ell=1}^{k} \sum_{j=1}^{\ell}\left(\begin{array}{c}
\ell-1 \\
j-1
\end{array}\right)(-1)^{j}(2 j-1)^{k-1} \log \Gamma_{\ell}\left(\frac{1}{2}\right) .
\end{aligned}
$$

\footnotetext{
${ }^{2} \mathrm{~A}$ generalization of the multiple gamma function as defined here, already introduced by Barnes in [2], occurs in Shintani's work on special values of Hecke $L$-functions [11].
} 
For $\chi$ a nontrivial Dirichlet character $\bmod N$

$$
L^{\prime}(1-k, \chi)+(\log N) L(1-k, \chi)=N^{k-1} \sum_{\ell=1}^{k} \sum_{r=1}^{N} \chi(r) P_{k, \ell}\left(\frac{r}{N}\right) \log \Gamma_{\ell}\left(\frac{r}{N}\right),
$$

where $P_{k, \ell}$ is defined in (11).

In particular, with the well known values

$$
\zeta(0)=-\frac{1}{2} \quad \zeta(-1)=-\frac{1}{12} \quad \zeta(-2)=0
$$

we easily get from from (12) that $\zeta^{\prime}(0)=-\frac{1}{2} \log 2 \pi$ as well as

$$
\begin{aligned}
& \zeta^{\prime}(-1)=-\frac{1}{36} \log 2+\frac{1}{6} \log \pi-\frac{2}{3} \log \Gamma_{2}\left(\frac{1}{2}\right) \quad \text { and } \\
& \zeta^{\prime}(-2)=-\frac{1}{14} \log \pi+\frac{8}{7} \log \Gamma_{2}\left(\frac{1}{2}\right)-\frac{8}{7} \log \Gamma_{3}\left(\frac{1}{2}\right) .
\end{aligned}
$$

\section{Epstein's zeta function and limit formula}

In order to prove Theorem 1 we shall employ a generalization of the Kronecker limit formula obtained by Epstein in 1903 [4]. Let $\mathcal{P}_{n}$ be the set of all $n \times n$ positive definite symmetric matrices. For $Q \in \mathcal{P}_{n}$ with $Q=\left(q_{i, j}\right)$ we have the associated quadratic form $Q(x)=\sum_{i, j} q_{i, j} x_{i} x_{j}$. The Epstein zeta function attached to $Q$ is defined for $\operatorname{Re}(s)>\frac{n}{2}$ by

$$
\zeta_{Q}(s)=\sum_{m \in \mathbb{Z}^{n}} Q(m)^{-s}
$$

It is well known [13] that $\zeta_{Q}(s)$ has meromorphic continuation to the entire s-plane with only a simple pole at $s=n / 2$ with residue

$$
\pi^{n / 2}|Q|^{-1 / 2} / \Gamma\left(\frac{n}{2}\right),
$$

where $|Q|=\operatorname{det} Q$, and that it satisfies the functional equation

$$
\pi^{-s} \Gamma(s) \zeta_{Q^{-1}}(s)=|Q|^{1 / 2} \pi^{s-n / 2} \Gamma\left(\frac{n}{2}-s\right) \zeta_{Q}\left(\frac{n}{2}-s\right) .
$$

In particular, $\zeta_{Q}(0)=-1$.

Suppose that $n \geq 2$. Then $Q$ can be written uniquely in the form

$$
Q=\left(\begin{array}{cc}
1 & 0 \\
-{ }^{t} x & 1
\end{array}\right)\left(\begin{array}{cc}
y^{-1} & 0 \\
0 & Y
\end{array}\right)\left(\begin{array}{cc}
1 & -x \\
0 & 1
\end{array}\right)
$$

with $y \in \mathbb{R}^{+}, x \in \mathbb{R}^{n-1}$ and $Y \in \mathcal{P}_{n-1}$. For $m \in \mathbb{R}^{n-1}$ write

$$
Q\{m\}=m \cdot x+i \sqrt{y Y(m)} \text {. }
$$

Clearly $Q\{m\} \in \mathcal{H}$, the upper half-plane, unless $m=0$. After applying the functional equation, Epstein's generalization of the Kronecker limit formula can be put in the form (cf. [15]): 
Proposition 4.1 (Epstein).

$$
\zeta_{Q}^{\prime}(0)=-2 \pi \sqrt{y} \zeta_{Y}\left(-\frac{1}{2}\right)-\log \left|(2 \pi)^{2} y \prod_{m \in \mathbb{Z}^{n-1}}^{+}\left(1-e^{2 \pi i Q\{m\}}\right)^{4}\right|,
$$

where the + superscript indicates that the product is taken over some choice of $\pm m$ and $m$ is non-zero.

Let us write $\zeta_{\left(Q_{1}, \ldots, Q_{r}\right)}(s)=\zeta_{Q}(s)$ if $Q$ is the block-diagonal matrix with $Q_{1}, \ldots, Q_{r}$ on the diagonal. The following simple product formula is an easy consequence of Proposition 4.1 obtained by arranging that the terms involving the Epstein zeta values $\zeta_{Y}\left(-\frac{1}{2}\right)$ cancel.

Corollary 4.1. Suppose that $Q \in \mathcal{P}_{n}$. Then

$$
\exp \left(\zeta_{(1, Q)}^{\prime}(0)-2 \zeta_{(4, Q)}^{\prime}(0)\right)=\frac{\pi^{2}}{4} \prod_{m \in \mathbb{Z}^{n}}^{+} \tanh ^{4}\left(\frac{\pi}{2} \sqrt{Q(m)}\right) .
$$

Before turning to the proof of Theorem 1, let us show how (1) follows from this Corollary. We employ the well known identities

$$
\zeta_{(1,1)}(s)=4 \zeta(s) L(s) \text { and } \zeta_{(4,1)}(s)=2\left(1-2^{-s}+2 \cdot 2^{-2 s}\right) \zeta(s) L(s)
$$

where for now we write $L(s)=L\left(s, \chi_{-4}\right)$. Using that $L(0)=\frac{1}{2}$ we get

$$
\zeta_{(1,1)}^{\prime}(0)-2 \zeta_{(4,1)}^{\prime}(0)=\log \frac{\pi}{4}+2 L^{\prime}(0)
$$

and hence by (18)

$$
e^{L^{\prime}(0)}=\sqrt{\pi} \prod_{m \in \mathbb{Z}^{+}} \tanh ^{2}\left(\frac{\pi m}{2}\right),
$$

from which (1) follows easily.

ProOF OF THEOREM 1. In order to prove (i) of Theorem 1, we use the identities

$$
\begin{aligned}
& \zeta_{(1,1,4,4)}(s)=2\left(1-\frac{1}{2^{s}}+\frac{4}{2^{3 s}}-\frac{16}{2^{4 s}}\right) \zeta(s) \zeta(s-1)+2 L(s) L(s-1) \\
& \zeta_{(4,1,4,4)}(s)=\left(1-\frac{3}{2^{s}}+\frac{10}{2^{2 s}}-\frac{32}{2^{4 s}}\right) \zeta(s) \zeta(s-1)+L(s) L(s-1) .
\end{aligned}
$$

For (19) see [3, p.381], while (20) follows from Jacobi's result for sums of 4 squares as in [17, p.202]. Thus we have

$$
\zeta_{(1,1,4,4)}^{\prime}(0)-2 \zeta_{(4,1,4,4)}^{\prime}(0)=-\frac{23}{6} \log 2+\log \pi-12 \zeta^{\prime}(-1)
$$

and upon using (14), (18) gives (i) of Theorem 1.

Turning now to the proof of (ii), classical results of Liouville [6, vol 9 p.273, p.257, p.421] imply, after some computation, the identities 


$$
\begin{aligned}
& \zeta_{(1,2,2,2,2,1)}(s)=4 \zeta(s-2) L(s)-4 \cdot 2^{-s} \zeta(s) L(s-2) \\
& \zeta_{(4,2,2,2,2,4)}(s)=8 \cdot 2^{-s} \zeta(s-2) L(s)-4 \cdot 2^{-2 s} \zeta(s) L(s-2) \\
& \zeta_{(1,2,2,2,2,4)}(s)=2 \zeta(s-2) L(s)-4 \cdot 2^{-2 s} \zeta(s) L(s-2)
\end{aligned}
$$

A routine calculation using that $L(-2)=-\frac{1}{2}$ gives

$$
\begin{aligned}
& A=\zeta_{(1,2,2,2,2,4)}^{\prime}(0)-2 \zeta_{(4,2,2,2,2,4)}^{\prime}(0)=\log \frac{\pi}{2}-2 L^{\prime}(-2)-7 \zeta^{\prime}(-2) \\
& B=\zeta_{(1,2,2,2,2,1)}^{\prime}(0)-2 \zeta_{(4,2,2,2,2,1)}^{\prime}(0)=\log \frac{\pi}{4}-2 L^{\prime}(-2)
\end{aligned}
$$

and hence we have by (15)

$$
e^{A-B}=2 e^{-7 \zeta^{\prime}(-2)}=2 \sqrt{\pi} \Gamma_{3}\left(\frac{3}{2}\right)^{8} .
$$

Now (ii) of Theorem 1 follows from (18).

We can derive (2) from the well known identity

$$
\zeta_{(1,2)}(s)-2 \zeta_{(4,2)}(s)=2\left(1-2 \cdot 2^{-s}\right) \zeta(s) L_{-8}(s),
$$

where $L_{D}(s)=L(s, \chi)$ for $\chi(\cdot)=\left(\frac{D}{.}\right)$. Now (18) gives, using that $L_{-8}(0)=$ 1 ,

$$
e^{L_{-8}^{\prime}(0)}=\frac{\pi}{2} \prod_{m \in \mathbb{Z}^{+}} \tanh ^{4}\left(\frac{\pi m}{\sqrt{2}}\right),
$$

from which (2) follows. A similar argument using

$$
\begin{aligned}
& \zeta_{(1,2,2,2)}(s)=4 \zeta(s-1) L_{8}(s)-2 \zeta(s) L_{8}(s-1) \text { and } \\
& \zeta_{(4,2,2,2)}(s)=2^{-s}\left(8 \zeta(s-1) L_{8}(s)-2 \zeta(s) L_{8}(s-1)\right),
\end{aligned}
$$

which follow from [6, vol 6. p.225], leads to the companion identity

$$
e^{-L_{8}^{\prime}(-1)}=\frac{\pi}{2}(\sqrt{2}-1) \prod_{m \in \mathbb{Z}^{3}}^{+} \tanh ^{4}\left(\frac{\pi|m|}{\sqrt{2}}\right) .
$$

Several more examples can easily be given, but the eventual appearance of cusp forms cannot be avoided.

\section{The $G L(n)$ eta-function at special points}

We are now ready to define a generalization of the Dedekind eta-function for $\operatorname{GL}(n)$ and give a formula of Chowla-Selberg type for it. For $Q \in \mathcal{P}_{n}$ with $n \geq 2$ consider the function

$$
\psi_{n}(s, Q)=\sum_{m \in \mathbb{Z}^{n-1}}^{*}\left(m \cdot x+i \sqrt{Y_{1}(m)}\right) Y_{1}(m)^{-s}
$$


where $Y_{1}=y Y$ with $x, y$ and $Y$ defined in (16), and the $*$ indicates that the sum is over $m$ such that the first nonzero entry of $m$ is positive. It can be verified that

$$
\psi_{2}(s, Q)=Y_{1}^{-s} \zeta(2 s-1)\left(x+i \sqrt{Y_{1}}\right) .
$$

A straightforward induction argument in $n$ shows that $\psi_{n}(s, Q)$ has a meromorphic continuation in $s$ and that $\psi_{n}(s, Q)$ is holomorphic at $s=0$. We define the $G L(n)$ eta function on $\mathcal{P}_{n}$ by

$$
\eta_{n}(Q)=e^{-\pi i \psi_{n}(0, Q)} \prod_{m \in \mathbb{Z}^{n-1}}^{+}\left(1-e^{2 \pi i Q\{m\}}\right),
$$

where $Q\{m\}$ is defined in (17). Clearly $\eta_{n}(\alpha Q)=\eta_{n}(Q)$ for $\alpha \in \mathbb{R}^{+}$, so $\eta_{n}$ is well defined on similarity classes of quadratic forms.

In case $n=2$ we can identify $\tau \in \mathcal{H}$ with

$$
Q_{\tau}=\left(\begin{array}{rr}
1 & 0 \\
-x & 1
\end{array}\right)\left(\begin{array}{cc}
y^{-1} & 0 \\
0 & y
\end{array}\right)\left(\begin{array}{cc}
1 & -x \\
0 & 1
\end{array}\right)
$$

so that for $g \in \mathrm{SL}(2, \mathbb{R})$ we have that $Q_{g^{-1} \tau}={ }^{t} g Q_{\tau} g$, where $g^{-1} \tau$ is the usual linear fractional action. It is easily checked using (22) that $\eta_{2}\left(Q_{\tau}\right)=$ $\eta(\tau)$, the classical eta-function defined in (5).

Clearly Epstein's limit formula in Proposition 4.1 can be written in the form

$$
(2 \pi)^{-2} e^{-\zeta_{Q}^{\prime}(0)}=y\left|\eta_{n}(Q)\right|^{4}
$$

It follows that $y^{1 / 4}\left|\eta_{n}(Q)\right|$ is invariant under $Q \mapsto{ }^{t} g Q g$ for any $g \in \operatorname{GL}(n, Z)$. It also follows that if $\mathcal{M}=\mathbb{R}^{n} / L$, where $L$ is a full lattice in $\mathbb{R}^{n}$, then

$$
\operatorname{det} \Delta_{\mathcal{M}}=y\left|\eta_{n}(Q)\right|^{4},
$$

where $Q=\left(\alpha_{i} \cdot \alpha_{j}\right)$ for $\left\{\alpha_{1}, \ldots, \alpha_{n}\right\}$ any integral basis for $L^{*}$, the lattice dual to $L$.

Turning finally to the Chowla-Selberg formula, we will assume for simplicity that $Q$ is an even unimodular quadratic form ${ }^{3}$, so that $8 \mid n$. Let

$$
r_{Q}(\ell)=\#\left\{m \in \mathbb{Z}^{n} ; \frac{1}{2} Q(m)=\ell\right\} .
$$

It is known from the theory of modular forms [5] that for $k=n / 2$

$$
r_{Q}(\ell)=\frac{n}{\left|B_{k}\right|} \sigma_{k-1}(\ell)+a(\ell)
$$

where $\sigma_{k-1}(\ell)=\sum_{d \mid \ell} d^{k-1}, B_{k}$ is the (usual) Bernoulli number and $f(\tau)=$ $\sum_{\ell \geq 1} a(\ell) e(\ell \tau)$ is a cusp form of weight $k$ for the full modular group. Let

$$
L(s, f)=\sum_{\ell \geq 1} a(\ell) \ell^{-s}
$$

\footnotetext{
${ }^{3}$ The same method applies to any even integral $Q$ but leads to a very complicated general formula.
} 
be the associated Dirichlet series, known to be entire. Define the rational numbers $C_{k}=\left(2^{k}-1\right)^{-1}$ and

$$
C_{k, \ell}=\frac{k C_{k}}{B_{k}} \sum_{j=1}^{\ell}\left(\begin{array}{c}
\ell-1 \\
j-1
\end{array}\right)(-1)^{j}(2 j-1)^{k-1} .
$$

Theorem 2. For $Q \in \mathcal{P}_{n}$ even unimodular we have

$$
y\left|\eta_{n}(Q)\right|^{4}=\frac{\lambda}{\pi} \prod_{\ell=1}^{k} \Gamma_{\ell}\left(\frac{1}{2}\right)^{C_{k, \ell}},
$$

where $k=n / 2$ and $\lambda=2^{C_{k}-2} e^{-L^{\prime}(0, f)}$.

Proof. By (26) we have the identity

$$
\zeta_{Q}(s)=\frac{n}{\left|B_{k}\right|} 2^{-s} \zeta(s) \zeta(s+1-k)+L(s, f) .
$$

Hence using that $\zeta(1-k)=-\frac{B_{k}}{k}$ we have

$$
\zeta_{Q}^{\prime}(0)=-\log \pi-\frac{k}{\left|B_{k}\right|} \zeta^{\prime}(1-k)+L^{\prime}(0, f)
$$

The proof is finished by applying (12) and (24).

For example, if $n=8$ and $Q$ is any even unimodular matrix in $\mathcal{P}_{8}$ then

$$
y\left|\eta_{8}(Q)\right|^{4}=\frac{2^{-29 / 15}}{\pi}\left(\frac{\Gamma_{1}\left(\frac{1}{2}\right) \Gamma_{3}\left(\frac{1}{2}\right)^{72}}{\Gamma_{2}\left(\frac{1}{2}\right)^{26} \Gamma_{4}\left(\frac{1}{2}\right)^{48}}\right)^{8} .
$$

If $n=16$ and $Q$ is any even unimodular matrix in $\mathcal{P}_{16}$ then

$$
y\left|\eta_{16}(Q)\right|^{4}=\frac{2^{-509 / 255}}{\pi}\left(\frac{\Gamma_{1}\left(\frac{1}{2}\right) \Gamma_{3}\left(\frac{1}{2}\right)^{73752} \Gamma_{5}\left(\frac{1}{2}\right)^{1948800} \Gamma_{7}\left(\frac{1}{2}\right)^{2257920}}{\Gamma_{2}\left(\frac{1}{2}\right)^{2186} \Gamma_{4}\left(\frac{1}{2}\right)^{595728} \Gamma_{6}\left(\frac{1}{2}\right)^{3037440} \Gamma_{8}\left(\frac{1}{2}\right)^{645120}}\right)^{16 / 17} .
$$

If $n=24$ then $L^{\prime}(0, f)=A_{Q} L^{\prime}\left(0, \eta^{24}\right)$ where $A_{Q} \in \mathbb{Q}$. It is a consequence of Siegel's main theorem [12] that

$$
\sum_{Q} w_{Q}^{-1} A_{Q}=0
$$

where the sum is over representatives of the genus of even unimodular matrices in $\mathcal{P}_{24}$ and $w_{Q}=\#\left\{g \in \mathrm{GL}(n, \mathbb{Z}) ;{ }^{t} g Q g=Q\right\}$. A similar result holds in general.

We end by remarking that there might be some interest in the further study of the general eta-function, including its analytic properties on various restrictions of $\mathcal{P}_{n}$ and its transformation properties under appropriate subgroups of $\mathrm{SL}(n, \mathbb{Z})$. 


\section{References}

[1] V. AdAmchiK, Multiple Gamma Function and Its Application to Computation of Series. preprint.

[2] E. W. BARNES, On the theory of the multiple Gamma function. Cambr. Trans. 19, (1904) 374-425.

[3] J. Elstrodt, F. Grunewald, AND J. Mennicke, Groups acting on hyperbolic space. Harmonic analysis and number theory. Springer Monographs in Mathematics. Springer-Verlag, Berlin, 1998.

[4] P. EPSTEIN, Zur Theorie allgemeiner Zetafunktionen. Math. Ann. 56, (1903), 615-644.

[5] E. HeCKE, Analytische Arithmetik der positive quadratischen Formen, (1940) \# 41 in Mathematische Werke.

[6] M.J. LiouviLle, Journal de Mathématiques Pure et Appliquées

[7] S. Minakshisundaram, AND A. Pleijel, Some properties of the eigenfunctions of the Laplaceoperator on Riemannian manifolds. Canadian J. Math. 1, (1949). 242-256.

[8] J. Dufresnoy, AND CH. Pisot, Sur la relation fonctionnelle $f(x+1)-f(x)=\varphi(x)$, Bull. Soc. Math. Belg. 15 (1963), 259-270.

[9] P. SARNAK, Determinants of Laplacians; heights and finiteness. Analysis, et cetera, 601-622, Academic Press, Boston, MA, 1990.

[10] S. SelberG,, S. ChOWla, On Epstein's zeta-function. J. Reine Angew. Math. 227 (1967), 86-110.

[11] T. SHINTANI, On special values of zeta functions of totally real algebraic number fields. Proceedings of the International Congress of Mathematicians (Helsinki, 1978), pp. 591-597, Acad. Sci. Fennica, Helsinki, 1980.

[12] C. L. SiEgEL, Über die analytische Theorie der quadratischen Formen. Ann. of Math. (2) 36 (1935), no. 3, 527-606. [in Gesammelte Abhandlungen]

[13] C. L. SIEGEL, Lectures on advanced analytic number theory. Notes by S. Raghavan. Tata Institute of Fundamental Research Lectures on Mathematics, No. 23 Tata Institute of Fundamental Research, Bombay 1965.

[14] H. M. SRIVASTAVA,, J. CHOI, Series associated with the zeta and related functions. Kluwer Academic Publishers, Dordrecht, 2001.

[15] A. TERRAS, Bessel series expansions of the Epstein zeta function and the functional equation. Trans. Amer. Math. Soc. 183 (1973), 477-486.

[16] I. VARDI, Determinants of Laplacians and multiple gamma functions. SIAM J. Math. Anal. 19 (1988), no. 2, 493-507.

[17] B. A. VenKov, Elementary number theory. Translated from the Russian and edited by Helen Alderson Wolters-Noordhoff Publishing, Groningen 1970.

[18] M.-F. VignéRAS, L'équation fonctionnelle de la fonction zêta de Selberg du groupe modulaire $\operatorname{PSL}(2, \mathbf{Z})$,in Journées Arithmétiques de Luminy , 235-249, Astérisque, 61 , Soc. Math. France, Paris, 1979.

UCLA Mathematics Dept.

Box 951555

Los Angeles, CA 90095-1555

E-mail : duke@math.ucla.edu

UCSB Mathematics Dept.

Santa Barbara, CA 93106

Current address: ETH, Mathematics Dept.

CH-8092, Zürich, Switzerland

E-mail: ozlem@math.ethz.ch 